\title{
Electronic Structure of Semiconducting Nanotubes Adsorbed on Metal Surfaces
}

\author{
Susumu Okada and Atsushi Oshiyama \\ Institute of Physics and Center for Computational Sciences, University of Tsukuba, Tsukuba 305-8571, Japan
}

(Received 18 February 2005; published 9 November 2005)

\begin{abstract}
A total-energy electronic-structure calculation is performed to explore energetics and electronic structures of nanotubes adsorbed on metal surfaces. We find that the charge transfer from metal surfaces to the nanotubes takes place depending on both the electronic structures of the adsorbed nanotubes and the work functions of the metal surfaces. In addition, we also find a substantial hybridization between the electron states of metal atoms and those of the nanotubes, which results in the metal-induced inhomogeneous charge distribution in the nanotubes.
\end{abstract}

DOI: 10.1103/PhysRevLett.95.206804

PACS numbers: 73.22.-f, 73.20.At, 73.40.Ns

In the last decade, there has been strong interest in and intensive research activity of the electronic and geometric properties of nanometer scale materials consisting of carbon atoms [1]. Among them, carbon nanotubes [2] have kept a premier position with their various interesting electronic properties which sensitively depend on the tiny differences of the atomic arrangements [3,4]. The peculiar electronic property opens the possibility of fabricating superior nanometer scale electronic devices [5] which consist presumably of hybrids of nanotubes and conventional materials. For instance, it has been demonstrated that the individual semiconducting nanotubes can work as field-effect transistors (FETs) in which nanotubes form contacts with various metal surfaces, such as $\mathrm{Pt}, \mathrm{Au}, \mathrm{Ca}, \mathrm{Al}$, etc. [5-8]. It was reported that the FETs exhibit different properties depending on the contact metal species; e.g., they exhibit $n$-type and $p$-type properties for $\mathrm{Ca}$ and Pd electrodes, respectively [7]. While the fabrication technology regarding nanotube is advancing steadily, little is known as to fundamentals of formation and properties of the hybrid structures, compared with the current stage of the semiconductor technology. In particular, the stability and properties of the interface between nanotubes and metals that have rarely been addressed before are of principal importance in the next generation of technology.

In complexes consisting of nanotubes and foreign atoms or molecules, it was demonstrated that the atoms or molecules can control geometric and electronic properties of the complexes due to a mixing between wave functions of nanotubes and those of the foreign atoms or molecules [9-13]. On the other hand, there are peculiar electron states which are localized near surfaces of matters (surface states). On one hand, these electron states are related to surface atomic reconstructions $[14,15]$, and on the other hand, they affect atomic and electronic structures of foreign species adsorbed on the surfaces. It is thus expected that each of the nanotube states and the metal-surface states plays its own role in atomic and electronic structures of a hybrid object, carbon nanotubes on conventional metals.
The purpose of this Letter is to unravel the interplay between the tube-origin and surface-origin electron states in determining stability and properties of nanotubes attached to metal surfaces. We here take a semiconducting $(10,0)$ nanotube adsorbed on the metal surfaces which is considered to be a structural model of the contact between nanotubes and metal electrodes. For the metal surfaces, we consider aluminum (100) and calcium (100) surfaces to elucidate the salient characteristics of these hybrid structures. We have found that the stability and the properties of the nanotube-metal contact strongly depend on metal species. It is in contrast to conventional semiconductor-metal contacts in which the Fermi level is virtually pinned due to intrinsic interface states such as metal-induced gap states, causing the band lineup of the two materials to be insensitive to metal species. More specifically, we have found that in the energy-minimized geometries the Fermi level is located just below the bottom of the $\pi^{*}$ band of the $(10,0)$ nanotube on $\mathrm{Al}(100)$ surfaces, while the Fermi level crosses several $\pi^{*}$ bands of the nanotube on the $\mathrm{Ca}(100)$ surfaces, indicating the electron transfer from the Ca surfaces to the nanotube. The calculated number of electrons transferred to the semiconducting nanotube is $5.2 \times$ $10^{6} \mathrm{~cm}^{-1}$. We also find that the transferred electrons distribute in the metal side of the tube compared with the opposite side, resulting from the mixing between the tubeorigin and the metal-origin electron states. Such hybridization is also found in a much enhanced way for the nanotube on $\mathrm{Al}(100)$ surfaces, contributing even to the binding of the tube. This results in the distance of $3.0 \AA$ between the nanotube and the $\mathrm{Al}$ surface, substantially shorter than the corresponding value of $4.5 \AA$ for the Ca surface.

All calculations have been performed based on the density functional theory $[16,17]$. We use the local density approximation to treat the exchange-correlation energy of interacting electrons $[18,19]$. Norm-conserving pseudopotentials with separable forms are adopted to describe the electron-ion interaction $[20,21]$. The valence wave functions are expanded in terms of a plane-wave basis set with the cutoff energy of $45 \mathrm{Ry}$. The conjugate-gradient minimization scheme is utilized for the electronic-structure 
calculation [22]. In the present study, we choose (100) surfaces of $\mathrm{Al}$ and $\mathrm{Ca}$ as representatives of metal surfaces and clarify salient characteristics of the semiconducting nanotube adsorbed on the metal surfaces. The $\mathrm{Al}$ and $\mathrm{Ca}$ surfaces are simulated by repeating slab models with 7 and 5 atomic layers, respectively. Each slab is separated by $20 \AA$-vacuum regions to analyze the electron states and energetics of the carbon nanotubes adsorbed on the surface. Here we place semiconducting $(10,0)$ nanotubes on the metal surfaces. Each nanotube is separated in the lateral direction by $6 \AA$ or larger to simulate the characteristics of the individual nanotubes on metals. We impose commensurability conditions between the one-dimensional periodicity of the nanotube and lateral periodicity of metal surfaces. Consequently, a unit cell along the tube axis that is parallel to the surfaces contains double periodicity of the zigzag nanotubes, and the tube is elongated in the direction by about 3\% [23]. Integration over two-dimensional Brillouin zone is carried out using eight $\boldsymbol{k}$ points.

Figures 1(a) and 1(b) show binding energies of the semiconducting $(10,0)$ nanotube on $\mathrm{Ca}(100)$ and $\mathrm{Al}(100)$ surfaces, respectively, as a function of the distance between a wall of the nanotube and the topmost surface atomic position. We find that the optimum spacing for $\mathrm{Al}(100)$ and $\mathrm{Ca}(100)$ surfaces is about 3.0 and $4.5 \mathrm{~A}$, respectively. Calculated absorption energies for $\mathrm{Al}$ and $\mathrm{Ca}$ are 3.21 and $0.47 \mathrm{eV}$, respectively, showing drastic difference caused by chemical-element difference of metals. Large absorption energy for the $\mathrm{Al}$ surface is due to the substantial hybridization between electron states of the nanotube and the $\mathrm{Al}$ surface, whereas the nanotube is bound to the Ca surface due to rather weak electrostatic and dispersive attractive interactions (see below).

Next, we focus on the electronic structures of the nanotube adsorbed on the metal surfaces. Figures 2(a) and 2(b) show the density of states (DOS) for the $(10,0)$ nanotube adsorbed on $\mathrm{Ca}(100)$ [tube/Ca] and on $\mathrm{Al}(100)$ [tube/ $\mathrm{Al}$ ] surfaces, respectively. For this calculation, we take 192 and $240 \boldsymbol{k}$ points for $\mathrm{Ca}$ and $\mathrm{Al}$ surfaces, respectively, in the integration over the two-dimensional Brillouin zone. We have examined the character of each electron state and
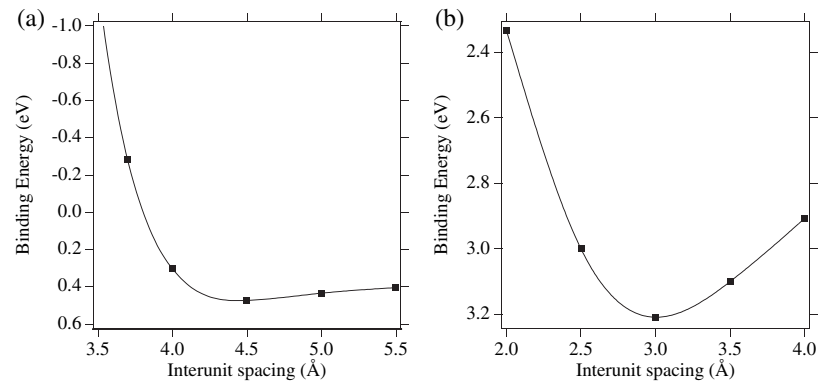

FIG. 1. Total energies of the semiconducting $(10,0)$ nanotubes on the (a) $\mathrm{Ca}(100)$ and (b) $\mathrm{Al}(100)$ surfaces, respectively, as a function of the distance between the nanotube wall and the topmost surface atomic position. The line is a guide for the eyes. found that the character of the $\pi$ and $\pi^{*}$ states of the nanotubes is more or less preserved, as is evidenced in the van Hove singularity shown by the arrows in Fig. 2. In the nanotube adsorbed on the $\mathrm{Ca}$ surface, we have found that the Fermi level is located by $0.2 \mathrm{eV}$ above the bottom of the conduction band of the $(10,0)$ nanotube and crosses several unoccupied $\pi$ electron bands. Thus, charge transfer from the $\mathrm{Ca}$ surface to the nanotube takes place. This results explain the recent experimental result that the nanotubes on the $\mathrm{Ca}$ contact electrode can work as an $n$-type FET [7]. Figure 3(a) shows the difference between the charge density of the nanotube on the surface and the sum of the charge densities of the isolated nanotube and of the tube-free Ca surface $\left(\Delta \rho=\rho_{\mathrm{NT}+\mathrm{Ca}}-\rho_{\mathrm{NT}}-\rho_{\mathrm{Ca}}\right)$. It is clear that electrons are transferred mainly from the $s$ state of the Ca to the $\pi$ state of the nanotube. Moreover, diminished charge of the $\mathrm{Ca}$ slab is mainly distributed around the interface region between the nanotube and the surface. Accumulated charges are distributed near the bottom of the nanotube facing the surface. The calculated amount of electrons injected into the nanotube is $5.2 \times$ $10^{6} \mathrm{~cm}^{-1}$. This charge transfer also affects the energy gap between the bottom of the conduction band and the top of the valence band of the pristine nanotube ( $E_{11}$ gap). The $E_{11}$ gap is wider by $20 \mathrm{meV}$ than that of the isolated $(10,0)$ nanotube.

Although the distance between the wall of the nanotube and the $\mathrm{Ca}$ surface is rather large, we observe sizable hybridization between $\mathrm{Ca}$ orbitals and nanotube orbitals.
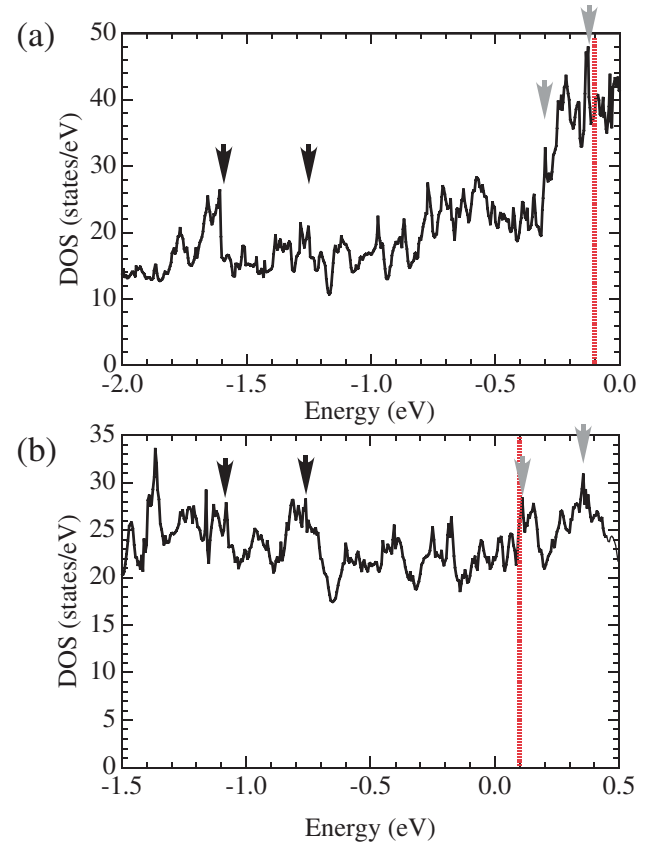

FIG. 2 (color). Density of states for the $(10,0)$ nanotube adsorbed on (a) $\mathrm{Ca}(100)$ and (b) $\mathrm{Al}(100)$ surfaces. The vertical dotted line denotes the Fermi level. The black and gray arrows indicate the electron states originated from the $\pi$ and $\pi^{*}$ states of the nanotubes, respectively. 
(a)

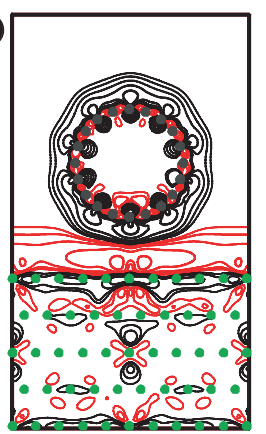

(b)

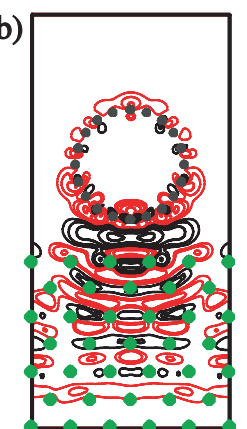

(c)

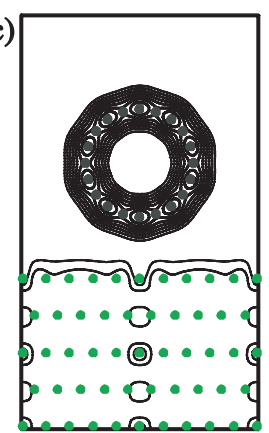

(d)

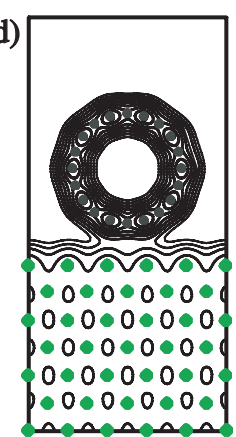

FIG. 3 (color). Contour plots of the accumulated and diminished charges for the $(10,0)$ nanotube on (a) the Ca(100) surfaces and (b) the $\mathrm{Al}(100)$ surfaces. Black and red contours denote the negatively charged and positively charged areas. Contour plots of valence total charge densities for (c) the $(10,0)$ nanotube on the $\mathrm{Ca}(100)$ surface and (d) the $(10,0)$ nanotube on the $\mathrm{Al}(100)$ surface. $\mathrm{Gray}$ and green circles denote the $\mathrm{C}$ and metal $(\mathrm{Ca}$ or $\mathrm{Al}$ ) atoms, respectively. Each contour represents twice (or half) the density of the adjacent contour lines.

From the wave function analysis at the $\Gamma$ point, we find that the doubly degenerate $\pi$ and $\pi^{*}$ states of the nanotube slightly split into two corresponding states at the $\Gamma$ point. The splitting energy is about a few $\mathrm{meV}$ for both the highest $\pi$ and the lowest $\pi^{*}$ bands. This result indicates that sizable hybridization between $\pi$ states of the nanotube and $s$ states of the metal takes place. Indeed, we find that the electron states around the Fermi level exhibit hybridized character distributing not only in the region of Ca but also on the nanotube to some extent [Fig. 4(a)].

Al surfaces provide other features that are in contrast to the Ca surfaces. We have found that the Fermi level is located just below the bottom of the conduction band of the $(10,0)$ nanotube which is adsorbed on the $\mathrm{Al}$ surface [Fig. 2(b)]. This difference from the Ca surface is partly
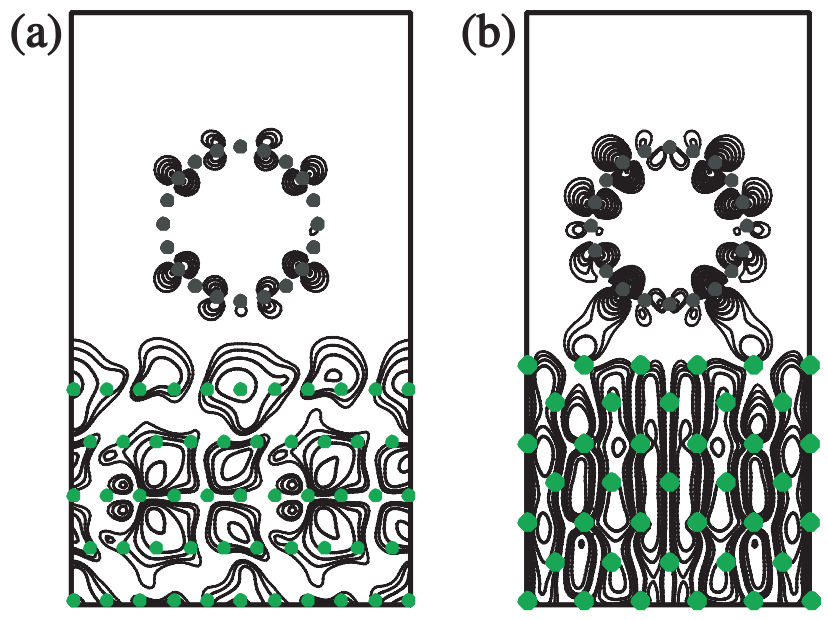

FIG. 4 (color). Contour plots of squared wave functions of electron states near the Fermi level. (a) An occupied state of the tube/Ca system located at about $0.2 \mathrm{eV}$ below the Fermi level. (b) An unoccupied state of the tube/Al system located at $0.07 \mathrm{eV}$ above the Fermi level. Gray and green circles denote the $\mathrm{C}$ and metals $(\mathrm{Ca}$ or $\mathrm{Al})$ atoms, respectively. Each contour represents twice (or half) the density of the adjacent contour lines. due to the different work functions: The work function of $\mathrm{Al}$ is larger than that of $\mathrm{Ca}$ by $1 \mathrm{eV}$. Owing to this larger work function, the charge transfer from the metal surface to the nanotube does not take place for the Al surface. However, adsorption of the nanotube results in the charge redistribution in both $\mathrm{Al}$ surfaces and the nanotube. In Fig. 3(b), the difference between the charge density of the nanotube on the Al surface and the sum of the charge densities of the isolated nanotube and of the tube-free $\mathrm{Al}$ surface is shown. It is clear that electrons are transferred mainly from the $\pi$ orbital of the nanotube and the $p$ orbital of the topmost $\mathrm{Al}$ atoms to the space between the tube and the $\mathrm{Al}$ surface. The distribution of the electron-rich region reflects the fact that the formation of weak covalent bonds between the nanotube and the surface $\mathrm{Al}$ atoms takes place. Valence electrons are evidently distributed between the nanotube and the topmost $\mathrm{Al}$ atoms [Fig. 3(d)]. This charge distribution exhibits different characteristics from that of the nanotube on Ca surfaces [Fig. 3(c)]. Indeed, we can see the remarkable hybridization between the $\pi$ states of the nanotube and the $p$ states of the topmost $\mathrm{Al}$ atoms [Fig. 4(b)], resulting in the formation of the weak covalent bond at the interface. For tube $/ \mathrm{Ca}, p$ orbitals of the metal are unavailable for the bond formation. The hybridization modulates the electronic structure of the nanotube. Indeed, the $E_{11}$ gap value decrease by $40 \mathrm{meV}$ from that of the isolated $(10,0)$ nanotube is in sharp contrast to the tube $/ \mathrm{Ca}$ system.

The present calculations clearly show that the charge redistribution near the tube-metal interface and also the lineup between the nanotube and the metal-surface electron states substantially depend on metal species, thereby providing rich variations of properties even with the same semiconductor tube. This is an interesting contrast to the case of conventional semiconductor-metal interfaces in which the band lineups of the two materials and thus the Schottky barrier heights are insensitive to metal species presumably due to intrinsic interface states [24]: when we take a semiconductor and examine a variety of metal 
contacts, the observed derivative of the Schottky barrier height with respect to the metal work function is 0.1 or less for elemental and $I I I-V$ semiconductors. This also causes difficulty in predicting and forming Ohmic contacts. In nanotube-metal interfaces, the band lineup is rather predictable from electron affinities and work functions of the two materials, as is shown here. This difference between nanotubes and conventional semiconductors is provisionally ascribed to the morphology of tube structures which modify the mixing with intrinsic states in metals.

In addition to the charge redistribution at the interface, we find substantial charge oscillation inside the nanotube and the $\mathrm{Al}$ slab. In particular, in the $\mathrm{Al}$ slab, we can clearly see the oscillation with the periodicity that corresponds to the spacing between adjacent atomic layers [Fig. 3(b)]. The electrons in both the nanotube and the Al slab undulate to screen charge redistribution caused by the hybridization of bond reformation near the interface.

In the $(10,0)$ nanotube on $\mathrm{Al}$, the Fermi level is located at just below the bottom of the conduction band of the nanotube so that the charge transfer from the surface to the nanotube does not take place. When thicker nanotubes or metallic nanotubes are adsorbed on the surface, however, electrons may be injected from the Al surface into the nanotube. This is because the energy gap between $\pi$ - and $\pi^{*}$-like states of the thick nanotube is narrower than that of the $(10,0)$ nanotube studied above. Furthermore, in the metallic nanotubes, the $\pi^{*}$ state touches the $\pi$ state at the particular wave number. In order to examine this possibility, we have performed the calculation for the $(9,0)$ metallic nanotube on the Al surfaces. Total-energy calculations reveal that the optimum distance between the nanotube and the $\mathrm{Al}$ surfaces is about $3.0 \AA$, which is the same as that for the $(10,0)$ nanotube on $\mathrm{Al}$ surfaces. We have indeed found that the lowest branch of the $\pi^{*}$ band of the nanotube crosses the Fermi level. The charge difference between the nanotube on the Al surface and the sum of each constituent unit, $\Delta \rho=\rho_{\mathrm{NT}+\mathrm{Al}}-\rho_{\mathrm{NT}}-\rho_{\mathrm{Al}}$, indicates the charge transfer from the Al surface to the nanotube. The calculated amount of the charge transfer in the nanotube is $1.1 \times 10^{6} \mathrm{~cm}^{-1}$. Besides the charge transfer, we also found the charge oscillation originated from the formation of weak covalent bond between the nanotube and the Al surface. Thus, the thicker or metallic nanotube adsorbed Al surfaces exhibits both ionic and covalent characters.

In summary, our calculations based on the density functional theory have clarified that the work function difference of metal surfaces changes the energetics and electronic properties of the nanotubes adsorbed on the surfaces. We found that the substantial charge transfer from $\mathrm{Ca}(100)$ surfaces to the $(10,0)$ nanotube takes place. In sharp contrast to the $\mathrm{Ca}$ surfaces, the charge transfer does not take place on the $\mathrm{Al}$ surfaces reflecting larger work function than the Ca surface.
This work was partly supported by ACT-JST, a special research project on nanoscience at the University of Tsukuba, NEDO project under the nanotechnology materials program, and a grant-in-aid for scientific research from the Japanese Ministry of Education. Computations were done at the SIPC, University of Tsukuba, and RCCS, Okazaki National Institute.

[1] M.S. Dresselhaus, G. Dresselhaus, and P.C. Eklund, Science of Fullerenes and Carbon Nanotubes (Academic Press, San Diego, 1996).

[2] S. Iijima, Nature (London) 354, 56 (1991).

[3] N. Hamada, S. Sawada, and A. Oshiyama, Phys. Rev. Lett. 68, 1579 (1992).

[4] R. Saito et al., Appl. Phys. Lett. 60, 2204 (1992).

[5] S. J. Tans, A. R. M. Verschueren, and C. Dekker, Nature (London) 393, 49 (1998).

[6] R. Martel et al., Appl. Phys. Lett. 73, 2447 (1998).

[7] Y. Nosho et al., Appl. Phys. Lett. 86, 073105 (2005).

[8] Y. Nosho et al., in Proceedings of the 25th Spring Meeting of the Japan Society of Applied Physics, Saitama, Japan, Extended Abstracts (Japan Society of Applied Physics, Tokyo, 2005), p. 1684.

[9] Y. Miyamoto et al., Phys. Rev. Lett. 74, 2993 (1995).

[10] S.-H. Jhi, S. G. Louie, and M. L. Cohen, Phys. Rev. Lett. 85, 1710 (2000).

[11] S. Okada, S. Saito, and A. Oshiyama, Phys. Rev. Lett. 86, 3835 (2001).

[12] M. Otani, S. Okada, and A. Oshiyama, Phys. Rev. B 68, 125424 (2003).

[13] W. Orellana, R. H. Miwa, and A. Fazzio, Phys. Rev. Lett. 91, 166802 (2003).

[14] K. C. Pandey, Phys. Rev. Lett. 47, 1913 (1981).

[15] K. Takayanagi et al., J. Vac. Sci. Technol. A 3, 1502 (1985).

[16] P. Hohenberg and W. Kohn, Phys. Rev. 136, B864 (1964).

[17] W. Kohn and L. J. Sham, Phys. Rev. 140, A1133 (1965).

[18] J. P. Perdew and A. Zunger, Phys. Rev. B 23, 5048 (1981).

[19] D. M. Ceperley and B. J. Alder, Phys. Rev. Lett. 45, 566 (1980).

[20] N. Troullier and J. L. Martins, Phys. Rev. B 43, 1993 (1991).

[21] L. Kleinman and D. M. Bylander, Phys. Rev. Lett. 48, 1425 (1982).

[22] O. Sugino and A. Oshiyama, Phys. Rev. Lett. 68, 1858 (1992).

[23] This elongation results in a narrowing of the gap energy between the top of valence band and the bottom of conduction band of the isolated $(10,0)$ nanotube by $50 \mathrm{meV}$. Also the central position in the gap is unchanged. Thus, the elongation does not affect discussions for the electronic properties of nanotubes on metal surfaces studied here. On the other hand, in order to clarify how the periodicity of the nanotube are modulated by the adsorption, further investigation is necessary.

[24] J. Tersoff, Phys. Rev. B 32, 6968 (1985), and references therein. 